\title{
Depression, anxiety and PTSD symptoms before and during the COVID-19 pandemic in the UK
}

K. S. Young ${ }^{1,2}$, K. L. Purves ${ }^{1,2}$, C. Hübel ${ }^{1,2,3}$, M. R. Davies ${ }^{1,2}$, K. N. Thompson 1 , S.

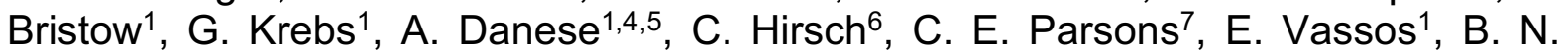
Adey $^{1}$, S. Bright ${ }^{1}$, L. Hegemann ${ }^{1}$, Y. T. Lee ${ }^{1}$, G. Kalsi ${ }^{1,2}$, D. Monssen ${ }^{1,2}$, J. Mundy ${ }^{1,2}$, A. J. Peel ${ }^{1}$, C. Rayner ${ }^{1}$, H. C. Rogers ${ }^{1,2}$, A. ter Kuile ${ }^{1,2}$, C. Ward ${ }^{1}$, K. York ${ }^{1}$, Y. Lin ${ }^{1}$, A. B. Palmos ${ }^{1}$, U. Schmidt ${ }^{2,8}$, D. Veale ${ }^{6,9}$, T. R. Nicholson ${ }^{9,10}$, T. A. Pollak ${ }^{9,10}$, S. A. M. Stevelink $^{8}$, T. Moukhtarian ${ }^{1}$, A. R. Martineau ${ }^{11}$, H. Holt ${ }^{11}$, B. Maughan ${ }^{1}$, A. Al-Chalabi ${ }^{12}$, K Ray Chaudhuri ${ }^{12,13}$, M. P. Richardson ${ }^{2,12}$, J. R. Bradley ${ }^{14}$, P. F. Chinnery ${ }^{14,15}$, N. Kingston ${ }^{14,16}$, S. Papadia ${ }^{14,17}$, K. E. Stirrups ${ }^{14,16}$, R. Linger ${ }^{14,17}$, M. Hotopf ${ }^{2,8}$, T. C. Eley $^{1,2}$, G. Breen*1,2

${ }^{1}$ Social, Genetic and Developmental Psychiatry Centre, Institute of Psychiatry, Psychology \& Neuroscience, King's College London, London, SE5 8AF, UK.

2 NIHR Maudsley Biomedical Research Centre, King's College London, UK.

${ }^{3}$ National Centre for Register-based Research, Department of Economics and

Business Economics, Aarhus University, Aarhus, Denmark

4 Department of Child \& Adolescent Psychiatry, Institute of Psychiatry, Psychology \& Neuroscience, King's College London, London SE5 8AF, United Kingdom

${ }^{5}$ National and Specialist CAMHS Trauma, Anxiety, and Depression Clinic, South London and Maudsley NHS Foundation Trust, London, UK

${ }^{6}$ Department of Psychology, Institute of Psychiatry, Psychology and Neuroscience, King's College London

${ }^{7}$ Interacting Minds Center, Department of Clinical Medicine, Aarhus University, Aarhus, Denmark

${ }^{8}$ Department of Psychological Medicine, Institute of Psychiatry, Psychology \&

Neuroscience, King's College London, London, UK

${ }^{9}$ South London and Maudsley NHS Foundation Trust

10 Section of Neuropsychiatry, Department of Psychosis Studies, Institute of

Psychiatry, Psychology and Neuroscience, King's College London, London, United Kingdom

${ }^{11}$ Blizard Institute, Barts and The London School of Medicine and Dentistry, Queen Mary University of London, London E1 2AT. 
${ }^{12}$ Department of Basic and Clinical Neuroscience, Institute of Psychiatry, Psychology and Neuroscience, King's College London, London, United Kingdom

13 Parkinson Foundation Centre of Excellence, King's College and King's College Hospital

14 NIHR BioResource and NIHR Cambridge Biomedical Research Centre, Cambridge University Hospitals NHS Foundation, Cambridge Biomedical Campus, Cambridge CB2 OQQ

${ }^{15}$ Department of Clinical Neurosciences and MRC Mitochondrial Biology Unit, University of Cambridge, Cambridge Biomedical Campus, Cambridge CB2 0QQ ${ }^{16}$ Department of Haematology, University of Cambridge, Cambridge Biomedical Campus, Cambridge, UK

${ }^{17}$ Department of Public Health and Primary Care, University of Cambridge, Cambridge Biomedical Campus,

Cambridge CB2 OQQ, UK

Corresponding author: Gerome Breen; address: Social, Genetic and Developmental Psychiatry Centre, Memory Lane, Camberwell, London SE5 8AF; email: gerome.breen@kcl.ac.uk; phone: +44 (0)20 78480409 


\section{Abstract}

Background: The COVID-19 pandemic is a novel population-level stressor. As such, it is important to examine pandemic-related changes in mental health and to identify which individuals are at greatest risk of worsening symptoms.

Methods: Online questionnaires were administered to 34,465 individuals in the UK, recruited from existing cohorts or via social media. Around one third $(n=12,718)$ with prior diagnoses of depression or anxiety completed pre-pandemic mental health assessments, allowing prospective investigation of symptom change. We examined changes in depression, anxiety and PTSD symptoms using prospective, retrospective and global ratings of change assessments. We also examined the effect of key risk factors on changes in symptoms.

Outcomes: Prospective analyses showed small decreases in depression (PHQ-9: .43 points) and anxiety symptoms (GAD-7: -.33 points), and increases in PTSD symptoms (PCL-6: .22 points). Conversely, retrospective analyses demonstrated large significant increases in depression (2.40 points) and anxiety symptoms (1.97 points) and $55 \%$ reported worsening mental health since the beginning of the pandemic on a global change rating. Using both prospective and retrospective symptom measures, regression analyses demonstrated that worsening depression, anxiety and PTSD symptoms were associated with i) prior mental health diagnoses, ii) female gender; iii) young age, and iv) unemployed or student status.

Interpretation: We highlight the effect of prior mental health diagnoses on worsening mental health during the pandemic and confirm previously-reported sociodemographic risk factors. Discrepancies between prospective and retrospective measures of changes in mental health may be related to recall bias underestimating prior symptom severity. 


\section{Research in context}

\section{Evidence before this study}

We conducted a literature search (PubMed, Scopus) with the terms "mental*" or "psychiatr" and "covid" or "coronavirus" published before 8th February 2021. This resulted in 4,573 unique references, but only 15 longitudinal studies examining changes in symptoms of mental health conditions from before to during the COVID19 pandemic. Results to date are mixed. Some studies found increases in mental distress, some found increases in either depression or anxiety, and others saw no observable change in symptoms.

Examining individual-level risk factors, heightened vulnerability to worsening mental health during the pandemic has been demonstrated among young people, females, individuals with lower incomes/financial problems and among health care or key workers. Only one previous study used a large sample with prior mental health diagnoses to examine changes in anxiety and depression. This study showed that having a prior mental health diagnosis was associated with higher levels of perceived worsening of mental health but, when examining actual prospectively measured symptoms, a prior mental health diagnosis was actually associated with a lower likelihood of symptom worsening, compared to no prior diagnosis. This discrepancy across measures requires further investigation in order to understand the nature of changing mental health during the COVID-19 pandemic.

\section{Added value of this study}

This study prospectively examined changes in symptoms of depression, anxiety and PTSD in a large UK-based sample of individuals with prior depression or anxiety. Analyses were supplemented with data from additional cohorts to examine individual difference risk factors with greater statistical power. Inclusion of both prospectively measured and retrospectively estimated changes in symptoms, as well as ratings of perceived change in mental health, allowed closer examination of discrepancies in subjective experience versus actual objective change in symptoms.

Consistent with prior reports, we observed high levels of reported worsening of mental health. Examination of prospective symptom change, however, showed small decreases in depression and anxiety and small increases in symptoms of PTSD. Exploring the discrepancy between perceived and prospectively measured symptom change, we observed that retrospective estimates of pre-pandemic depression and anxiety underestimated symptom severity (i.e. recalled symptoms were less severe than prospectively measured symptoms). This is likely to contribute to subjective experiences of worsening mental health. However, we also found that a range of prior mental health diagnoses, including eating disorders and co-morbid depression and anxiety, were associated with greater risk for worsening mental health during the pandemic, using either prospective or retrospective measures. We also confirm previous findings of greater risk for worsening symptoms among women, younger people and individuals who are students or are unemployed.

\section{Implications of all the available evidence}

This work highlights the increased likelihood of worsening mental health during the pandemic among individuals with pre-existing mental health diagnoses. It also replicates findings from other work demonstrating the vulnerability of young people, women, and 
people who are students or are unemployed. Additionally, discrepancies in estimated symptom change across prospective and retrospective measures highlight the importance of using prospectively collected data to examine longitudinal changes. 


\section{Introduction}

The COVID-19 pandemic has resulted in a globally experienced set of interrelated stressful life events. These stressors are likely to take a toll on mental health in both the general population and those infected with SARS-CoV- $2^{1}$. Stressful life events are known to heighten the risk for onset or worsening of depression, anxiety and stress-related conditions (i.e., acute/post-traumatic stress disorders) ${ }^{2-4}$. At the same time, measures put in place to mitigate the spread of the virus diminish access to sources of support, such as social contact and routine health care ${ }^{5,6}$, likely further contributing to the mental health burden. Establishing which groups are most adversely affected by the COVID-19 pandemic is a key research priority ${ }^{1}$.

Reports from longitudinal studies including pre-pandemic measures of mental health vary considerably. A UK sample of $\sim 53,000$ adults ${ }^{6}$ found elevated levels of mental distress (12-item General Health Questionnaire). A UK birth cohort of young adults (mean age 27 years, $\mathrm{N}=2,973)^{7}$ found increased anxiety, but not depression. $\mathrm{A}$ case-control sample in the Netherlands $(N=1,517)^{8}$ of individuals with depression, anxiety or obsessive-compulsive disorder (OCD) found increased depression but not anxiety. At the same time, other studies have found no observable changes in mental wellbeing, including a Dutch sample measuring change in anxiety and depression symptoms $(\mathrm{N}=3,983)^{9}$, and a US sample examining change in psychological distress $(\mathrm{N}=1,870)^{10}$.

Examining individual-level risk factors, longitudinal studies have demonstrated heightened vulnerability to worsening mental health during the pandemic among young people ${ }^{6}$, females ${ }^{6,7}$ and individuals living in socio-economic adversity ${ }^{7}$. Several smaller studies $(n<500)$ of young people have demonstrated worsening symptoms in groups observed in the US, Italy, India, Switzerland and China ${ }^{11-15}$. One study of older adults in the UK aged 55 years or over $(n=3,281)$ showed an increase in reporting of mild depression and anxiety symptoms, but no change in the frequency of reporting of moderate symptoms ${ }^{16}$.

In relation to prior mental health diagnoses, one birth cohort sample demonstrated that worsening depression and anxiety symptoms during the pandemic were associated with a history of major depressive disorder, generalised anxiety disorder or eating disorders ${ }^{7}$. However, in a case-control study of individuals with anxiety, depression or OCD, greater increases in prospectively assessed mental health symptoms were observed among individuals with no pre-existing mental health diagnoses, compared to those with diagnoses ${ }^{8}$. Furthermore, in those with the most chronic and severe mental health diagnoses, slight decreases in symptom severity were observed ${ }^{8}$. In contrast, this study also showed that individuals with prior mental health diagnoses reported a greater perceived impact of the pandemic on their mental wellbeing, compared to those without diagnoses ${ }^{8}$. Further examination of the relationship between perceived changes in distress and changes in symptom severity, will be critical to understanding vulnerabilities to worsening mental health during a pandemic.

Perceived changes in mental health in the absence of prospectively measured symptom change may be related to memory biases widely observed in depression and anxiety ${ }^{17}$. Longitudinal epidemiological studies indicate that rates of depression 
and anxiety disorder diagnoses are approximately double when measured prospectively compared to retrospectively ${ }^{18}$. In addition, 12-month recall accuracy of depression symptoms during a clinical trial ranged from $55 \%-95 \%$, depending on the symptom ${ }^{19}$. It is therefore plausible that lack of recall of prior symptoms contributes to the perception of worsening mental health, even if prospective measures of symptoms show no change.

The first aim of the current study was to examine changes in depression, anxiety and PTSD symptoms from before to during the COVID-19 pandemic. To examine discrepancies across measurement approaches we investigated symptom change in three ways: 1) prospectively measured symptom change; 2 ) retrospectively estimated symptom change; and 3 ) perceived changes in symptoms. Our second aim was to examine whether pre-existing mental health diagnoses and demographic factors (gender, age, ethnicity, employment status) were associated with greater change in symptoms of major depressive disorder (MDD), generalised anxiety disorder (GAD) and PTSD from before to during the pandemic.

\section{Methods}

Data were examined from two longitudinal online questionnaire studies: 1) the COVID-19 Psychiatry and Neurological Genetics study (COPING), and 2) the Repeated Assessment of Mental Health in Pandemics (RAMP; https://rampstudy.co.uk) study. In analyses presented below, the COPING study is divided into two cohorts, 'GLAD' and 'NBR' and are compared alongside the RAMP cohort. The COPING study division was based on whether participants were existing members of the Genetic Links to Anxiety and Depression study, who had completed prospective pre-pandemic mental health assessments (GLAD cohort), or whether they were members of other NIHR BioResource studies (NBR cohort; see below). All samples were combined for regression analyses to maximise power to explore differential risk across demographic groups.

\section{Participants}

Recruitment for COPING and RAMP was conducted on a rolling basis, beginning in April 2020 (Figure 1). COPING participants were recruited from existing recontactable cohorts hosted by the NIHR BioResource. 12,718 individuals were from the GLAD study (34.6\% response rate). 13,096 individuals were from other NIHR Bioresource cohorts (19.3\% response rate), here referred to as 'NBR', including members of: i) the Eating Disorders Genetic Initiative (EDGI; $n=65)$; ii) the Irritable Bowel Disease cohort (IBD; $n=3,313$ ); and iii) general population cohorts ( $n=$ 9,718). GLAD and EDGI participants were originally recruited via social and traditional media campaigns, as well as through NHS organisations. IBD and general population cohorts were recruited via various means including advertisement in blood donation centres. RAMP study participants $(n=8,651)$ were recruited via social media advertising.

Eligibility criteria for both studies were aged $16+$ years and resident in the UK. GLAD eligibility criteria required either self-report of a previous depressive or anxiety disorder diagnosis, or meeting current DSM-5 criteria for depression or anxiety ${ }^{20}$. Ethical approval was granted by: i) NHS Health Research Authority, South West - 
Central Bristol Research Ethics Committee (20/SW/0078; COPING), and ii)

Psychiatry, Nursing and Midwifery Research Ethics Committee at King's College London (HR-19/20-18157; RAMP). Information sheets, consent forms and questionnaires were reviewed by the Feasibility and Acceptability Support Team for Researchers and the Service User Advisory Group.

\section{Measures}

All questionnaire data were acquired using Qualtrics survey software (Qualtrics, Provo, UT).

Symptoms of MDD, GAD and PTSD were assessed using the Patient Health Questionnaire (PHQ-9) ${ }^{21}$, Generalized Anxiety Disorder-7 scale (GAD-7) ${ }^{22}$ and abbreviated PTSD checklist (PCL-6) ${ }^{23}$, respectively. We examined differences in these measures in relation to prior mental health diagnoses and demographic factors of: gender, age, ethnicity and employment status (see Figure 1, Table S2 and Supplementary materials).

\section{Assessment timepoints}

Pre-pandemic symptoms (prospective): GLAD cohort participants completed prepandemic measures of MDD, GAD and PTSD at time of enrolment into the GLAD study (Sept 2018-Feb 2020).

Current symptoms during the pandemic: Participants completed PHQ-9, GAD-7 and PCL-6 scales at the point of enrolment into COPING or RAMP during the pandemic (April to September 2020; see Figure 1).

Pre-pandemic symptoms (retrospective): Immediately following current symptom assessment, participants completed a 'global rating of change' (see Supplementary materials ${ }^{24}$ ). Then, they completed retrospective estimates of pre-pandemic PHQ-9 and GAD-7 to assess recalled symptoms 'before the pandemic'.

\section{Statistical analyses}

Data on the PHQ-9, GAD-7 and PCL-6 were excluded if one or more items on each scale were not completed (missingness: 0.22\% PHQ-9; 0.17\% GAD-7; 0.41\% PCL$6)$. There were no exclusions made based on mental health diagnostic history or demographic factors (see Supplementary Materials for power calculation).

Change in symptoms: We first examined global change ratings in depression and anxiety. Next, Welch $t$-tests were used to assess differences in depression, anxiety and PTSD scores, as follows. Taking a prospective approach within the GLAD sample comparing symptoms before and during the pandemic assessing: i) mean symptom scores, and ii) binary threshold scores (proportions meeting clinically significant thresholds). Taking a retrospective approach we compared pre-pandemic symptom scores recalled during the pandemic with current symptom scores. Finally, to examine the association between prospective and retrospective measures of depression and anxiety symptoms, correlation analyses were conducted (GLAD sample only).

Individual differences in symptom change: Linear regression analyses included diagnostic (mental health diagnostic history) and demographic factors as explanatory variables, entered simultaneously into multivariable regression models. The first set 
of analyses were restricted to the GLAD sample and examined change in depression, anxiety and PTSD by controlling for prospectively measured prepandemic symptoms. The second set of analyses were conducted in the combined sample (and each sample individually) and examined change in depression and anxiety by controlling for retrospectively estimated pre-pandemic symptoms.

Diagnostic history variables were binary coded (present/absent), and demographic variables were coded categorically (see Supplementary materials). Associations with current depression, anxiety and PTSD symptoms were tested in separate models.

\section{Results}

Demographics are presented in Figure 1 and Table S2. Frequencies of prior mental health diagnoses were $98.8 \%$ in GLAD, 29.4\% in NBR and $58.1 \%$ in RAMP (Figure 2).

\section{Aim 1: Describing change in symptoms}

Perceived change in symptoms (all samples): Overall, $55.9 \%$ reported worsening depression (15.9\% much worse, $40.0 \%$ a little worse) and $54.0 \%$ reported worsening anxiety (16.7\% much worse, $37.3 \%$ a little worse). In the GLAD sample, frequencies of reported worsening were $62.8 \%$ and $62.0 \%$, for depression and anxiety, whereas, in NBR, these frequencies were $39.9 \%$ and $36.5 \%$ and, in RAMP, $70.4 \%$ and $68.9 \%$ (Table S3).

\section{Prospectively measured symptom change (GLAD only): Comparing} prospectively measured pre-pandemic symptoms to current symptoms, there was a small decrease in depression (PHQ-9; $M=-.43, S D=5.79, t(12110)=-8.15, p<$ $.0001)$ and anxiety (GAD-7; $M=-.33, S D=5.48, t(12125)=-6.67, p<.0001)$, and a small increase in PTSD (PCL-6; $M=.22, S D=5.69, t(11990)=4.30, p=<.0001$; Table 1). Although statistically significant, the observed changes were small in magnitude and standard deviations were large, indicating a high degree of variance. Comparing proportions of individuals meeting standardised cutoffs on each scale ( $\geq 10$ PHQ-9; $\geq 10$ GAD-7; $\geq 14$ PCL-6), there was a comparable pattern of effects. Proportions of individuals meeting thresholds for moderate depression reduced from $54.5 \%$ pre-pandemic to $51.8 \%$ during the pandemic, for moderate generalised anxiety disorder reduced from $40.6 \%$ to $38.9 \%$; and for probable PTSD increased from $56.4 \%$ to $57.9 \%$.

Retrospectively estimated symptom change (all samples): Comparing retrospectively estimated pre-pandemic symptoms to current symptoms demonstrated significant increases in PHQ-9 and GAD-7 scores overall $(t(34198)=$ $96.81, p<.0001 ; t(34323)=86.31, p<.0001)$ and in each individual sample (GLAD: $t(25019.6)=33.54, p<.0001 ; t(24656.4)=34.23, p<.0001 ; \operatorname{NBR}: t(24896.2)=$ $22.30, p<.0001 ; t(24674)=19.55, p<.0001$; RAMP: $t(16550.2)=41.38, p<.0001$; $t(16205)=39.00, p<.0001 ;$ Table 1$)$.

Correlation analyses (GLAD only): The correlations between pre-pandemic measures and retrospective estimates of PHQ-9 and GAD-7 were $r=.59$ [95\% Cl: $0.55-0.63$ ] and $r=.51$ [95\% Cl: $0.47-0.56]$, respectively. Thus, the shared variance was relatively low at $34.81 \%$ for $P H Q-9$ and $26.01 \%$ for GAD-7. Direct comparison of 
means demonstrated significantly lower scores for retrospective estimates, compared to prospective measures of both PHQ-9 $(t(12066)=61.17, p<.001)$ and GAD-7 $(t(12110)=57.16, p<.001$; Table S4). This suggests that evidence of worsening symptoms based on retrospective reporting may be related to underestimation of pre-pandemic symptom severity.

\section{Aim 2: Associations with change in symptoms}

\section{Prospectively measured symptom change (GLAD only)}

Regression analyses examined individual differences associated with change in depression, anxiety and PTSD symptoms from before to during the pandemic. In each model, levels of pre-pandemic symptoms were positively associated with levels of current symptoms, so analyses report findings after accounting for pre-pandemic symptoms. Higher levels of current depression symptoms were associated with prior diagnosis of: i) depression and anxiety, ii) depression only, iii) eating disorders, iv) obsessive-compulsive and related disorders (OCRDs), v) PTSD, vi) autism spectrum disorder (ASD) and vii) personality disorders. Higher levels of current anxiety symptoms were associated with prior diagnosis of: i) depression and anxiety, ii) eating disorders, iii) OCRDs, iv) PTSD, v) ASD and vi) personality disorders. Higher levels of current PTSD were associated with prior diagnosis of: i) eating disorders, ii) OCRDs, iii) PTSD, and iv) personality disorders (Figure 3, Table S5).

Compared to male gender, female gender was associated with higher levels of current anxiety and PTSD, but not depression. Compared to the 26-35 year old reference group: i) 16-25 year olds reported higher current levels of depression, anxiety and PTSD symptoms; ii) 36-45 year olds reported lower levels of PTSD symptoms; iii) 46-75 year olds reported lower depression, anxiety and PTSD symptoms; iv) 76+ years olds were not significantly different. Compared to White ethnicity, there were no significant effects of minority ethnic group status. Compared to being employed, being a student was associated with higher current levels of depression symptoms and being unemployed was associated with higher current levels of depression, anxiety and PTSD symptoms (Figure 3, Table S5).

\section{Retrospectively estimated symptom change (all samples)}

Samples were combined to maximise power to explore differences in symptoms based on diagnostic and demographic factors. Patterns of effects were largely consistent with the prospective model. There were three additional associations with prior mental health diagnoses: i) prior anxiety disorder only with higher depression and anxiety symptoms; ii) prior bipolar disorder only with higher depression symptoms; and iii) combined psychotic and bipolar disorder diagnosis with higher anxiety symptoms. Additionally, there were significant effects of female gender, nonbinary/self-defined gender and student status on depression symptoms and key worker status on anxiety symptoms (Table S6).

Results of regression analyses for individual samples (GLAD, NBR, RAMP) are presented in supplementary materials (Tables S7-S8). Overall, the direction of effects was similar across all models although effects reached statistical significance in some samples, but not others. 


\section{Discussion}

In a large UK study $(\mathrm{N}=34,465)$, approximately $55 \%$ reported experiencing worsening of depression and anxiety symptoms from before to during the COVID-19 pandemic. Around a third of this sample were part of a mental health cohort study (GLAD) who had completed pre-pandemic mental health measures. In GLAD, 63\% reported perceived worsening symptoms. However, prospectively measured changes in symptoms indicated marginal decreases in anxiety and depression and a marginal increase in PTSD symptoms. In contrast, comparisons based on retrospective estimates of pre-pandemic symptoms indicated recall of significantly lower symptoms prior to the pandemic compared to during the pandemic, likely contributing to the experience of perceived symptom worsening. Examining individual difference factors associated with risk for worsening symptoms, we observed significant effects of lifetime diagnosis of a number of mental health conditions, female gender, young age (16-25 years) and being a student or being unemployed. These effects were consistent when controlling either for prospectively measured or retrospectively estimated pre-pandemic symptoms.

\section{Discrepancies across methods of estimating symptom change}

Despite high levels of reported experiences of worsening of mental health, there were small $(<0.5$ points per scale) decreases in depression and anxiety symptoms, and a small (0.2 point) increase in PTSD symptoms in the GLAD sample. While statistically significant, these marginal changes in symptoms are not considered to be clinically meaningful, as reliable change is estimated to be around 7 points on the $\mathrm{PHQ}-9$ and 5 points on the GAD- $7^{25}$. Our findings are consistent with another COVID-19 mental health study showing no significant change in depression or anxiety symptoms among individuals with prior diagnosis of depression, anxiety or $\mathrm{OCD}^{8}$. As discussed below, various prior mental health diagnoses do appear to contribute to risk for worsening symptoms. However, marginal overall changes in symptoms suggest that there are as many individuals showing a decrease in symptoms as there are showing an increase from before to during the pandemic. It is plausible that for many, the stay-at-home lifestyle of the pandemic reduced daily sources of stress, such as social pressures or workplace challenges. A marginal overall decrease in symptoms of depression and anxiety may also be indicative of regression to the mean ${ }^{29}$ but the marginal increases in PTSD symptoms we observe are inconsistent with this explanation.

Increase in PTSD symptoms may occur via exposure to new traumatic stressors during the pandemic (e.g., life-threatening illness such as acute COVID-19 infection, domestic/other abuse), exacerbation of existing PTSD symptoms, or may reflect broader non-trauma related changes in mental distress. Further investigation of longitudinal PTSD symptom change in relation to experienced stressors would be important to distinguish these explanations.

We observed a discrepancy between high levels of perceived worsening of symptoms and no evidence of prospective symptom change. Prior work comparing PHQ-9 symptoms and global ratings of change noted similar discrepancies, attributed in part to global ratings being a more 'holistic' assessment, a lack of recall of prior symptoms and tendencies to underscore on PHQ-926. Our retrospective examination of symptom severity aligns with these findings, demonstrating 
significantly lower retrospective estimates compared to prospective measures. This underestimation of past symptom severity may contribute to a perceived worsening of mental health even without overall symptom increases.

Whereas prospective measures assessed 'past two week' symptoms, retrospective measures assessed 'how you usually felt before the pandemic'. This likely introduced error in the timeframes being compared, as well as potential overgeneralisation in retrospective reporting. In addition, retrospective estimates were completed after the global change rating, which may have introduced a confirmation bias. It is also possible that ratings of subjective changes in mental health capture short-term psychological distress, which may be a precursor to longer-term changes in symptomatology. Analysis of follow-up timepoints will be critical to determine longer-term changes in mental health during the COVID-19 pandemic.

\section{Risk factors: prior mental health diagnosis}

Regression analyses controlling for prospectively measured pre-pandemic symptoms demonstrated that a range of pre-existing mental health diagnoses were associated with risk for worsening depression, anxiety and PTSD. These included eating disorders, OCRDs, PTSD and personality disorders. Additionally, comorbid prior depression and anxiety diagnoses and ASD diagnosis were associated with worsening depression and anxiety. Despite differing estimates of pre-pandemic symptom levels using prospective versus retrospective measurements, regression models controlling for retrospective estimates identified a largely similar pattern of effects. Additional significant effects, with larger effect sizes, were observed in retrospective models for 'depression only' and 'anxiety only' diagnoses and bipolar disorder (depression symptoms only), potentially due to increased power to detect these effects in a larger sample.

\section{Risk factors: demographic variables}

We confirm prior observations demonstrating greater risk for worsening mental health among female and younger participants ${ }^{6,7,27}$, as well as in students and individuals who were unemployed. Female gender was associated with higher levels of anxiety and PTSD, but not depression symptoms. Younger age and being unemployed were both associated with higher levels of depression, anxiety and PTSD, whilst being a student was associated with higher levels of depression only. We observed no statistically significant effects of ethnicity, although it should be noted that these analyses were powered only to detect differences with a moderate effect size. Smaller effects may be present that were not observable in the present work, and examination of these should be a priority for future work. Combined factors of younger age and student/unemployment status may put young people at particular risk of worsening mental health during the pandemic.

\section{Limitations}

A primary limitation of this work is that convenience sampling resulted in a cohort overrepresented in females and individuals of White ethnicity ${ }^{28}$. Secondly, analyses examining prospectively measured changes in symptoms were available only in individuals with prior diagnosis of depression or anxiety (GLAD sample). The absence of an unaffected control group in these analyses may underestimate effect sizes 
because presence of one diagnosis is compared not against 'no diagnosis', but against individuals who do not have the diagnosis of interest. However, such comparisons do provide a stringent examination of the differential risk of a range of mental health diagnoses among groups of individuals who have prior experience of mental distress. Thirdly, reporting of prior mental health diagnoses is likely to be impacted by recall errors that bias towards under-reporting. Although certainly a limitation, this would likely reduce the power to detect differences based on diagnostic status with more individuals being misallocated to the 'no diagnosis' group and would more likely result in false negative than false positive effects.

\section{Conclusion}

In a large UK study combining 3 samples, we demonstrate that over half reported experiencing worsening mental health from before to during the COVID-19 pandemic. However, among a subsample of participants with prior depression or anxiety and prepandemic symptom measures, there was no evidence of clinically meaningful symptom change at the overall group level. Examining individual risk factors, prior mental health diagnosis, young age and unemployment was associated with worsening depression, anxiety and PTSD symptoms from before to during the COVID19 pandemic. In addition, female gender was associated with worsening symptoms of anxiety and PTSD, but not depression, and being a student was associated with worsening depression, but not anxiety or PTSD. These groups of individuals are likely to be in particular need of support to prevent worsening of mental health during the ongoing COVID-19 crisis.

\section{Contributors}

KSY, KLP, SBristow, CHuebel, MH, TCE and GB devised the study concept and all authors were consulted on measurement inclusion. KSY, KLP, CHuebel, MRD, KNT and GB wrote the analysis plan. Data was curated and analysed by KLP, CHuebel, MRD, KNT, LH, AJP, YL and HCR. Figures were produced by KLP and SBright. KSY wrote the first draft of the manuscript and all authors contributed to editing and commenting on the final version.

\section{Declaration of interests}

We declare no competing interests

\section{Acknowledgements}

We thank NIHR BioResource volunteers for their participation, and gratefully acknowledge NIHR BioResource centres, NHS Trusts and staff for their contribution. We thank the National Institute for Health Research, NHS Blood and Transplant, and Health Data Research UK as part of the Digital Innovation Hub Programme. This work was supported by the National Institute of Health Research (NIHR) BioResource Centre Maudsley and is part-funded by the National Institute for Health Research (NIHR) Biomedical Research Centre at South London and Maudsley NHS Foundation Trust and King's College London. Patient and public involvement groups and services were provided by the NIHR KCL-Maudsley Biomedical Research Centre. The views expressed are those of the author(s) and not necessarily those of 
the NHS, the NIHR or the Department of Health and Social Care. The RAMP study is supported in part by the King's Together Multi and Interdisciplinary Research Scheme (Rapid COVID-19 call, Round 1; Round 2). KSY is supported by MQ; Transforming Mental Health (MQF20/24). CH acknowledges funding from Lundbeckfonden (R276-2018-4581). TE and GB are part funded by a program grant from the UK Medical Research Council (MR/V012878/1). AAC is an NIHR Senior Investigator and AAC and GB part supported through EU Joint Programme Neurodegenerative Disease Research (JPND) projects through the following funding organisations under the aegis of JPND - www.jpnd.eu (United Kingdom, Medical Research Council (MR/L501529/1; MR/R024804/1). PFC is a Wellcome Trust Principal Research Fellow (212219/Z/18/Z) who receives support from the Medical Research Council Mitochondrial Biology Unit (MC_UU_00015/9).

\section{Data sharing}

Deidentified data included in analyses presented here is available from study authors on request. Analysis code will be made available at publication from: https://github.com/klpurves/PANCHANGE scripts

\section{References}

1. Holmes, E. A. et al. Multidisciplinary research priorities for the COVID-19 pandemic: a call for action for mental health science. The Lancet Psychiatry 7, $547-560$ (2020).

2. Daviu, N., Bruchas, M. R., Moghaddam, B., Sandi, C. \& Beyeler, A. Neurobiological links between stress and anxiety. Neurobiology of Stress 11, 100191 (2019).

3. Hammen, C. Stress and depression. Annu Rev Clin Psychol 1, 293-319 (2005).

4. Kilpatrick D. G. et al. National Estimates of Exposure to Traumatic Events and PTSD Prevalence Using DSM-IV and DSM-5 Criteria. Journal of Traumatic Stress 26, 537-547 (2013).

5. Danese, A. \& Smith, P. Debate: Recognising and responding to the mental health needs of young people in the era of COVID-19. Child and Adolescent Mental Health 25, 169-170 (2020).

6. Pierce, M. et al. Mental health before and during the COVID-19 pandemic: a 
longitudinal probability sample survey of the UK population. The Lancet Psychiatry 7, 883-892 (2020).

7. Kwong, A. S. F. et al. Mental health before and during COVID-19 in two longitudinal UK population cohorts. Br J Psychiatry 1-27 (2020) doi:10.1192/bjp.2020.242.

8. Pan, K.-Y. et al. The mental health impact of the COVID-19 pandemic on people with and without depressive, anxiety, or obsessive-compulsive disorders: a longitudinal study of three Dutch case-control cohorts. The Lancet Psychiatry $\mathbf{0}$, (2020).

9. van der Velden, P. G., Contino, C., Das, M., van Loon, P. \& Bosmans, M. W. G. Anxiety and depression symptoms, and lack of emotional support among the general population before and during the COVID-19 pandemic. A prospective national study on prevalence and risk factors. J Affect Disord 277, 540-548 (2020).

10. Breslau, J. et al. A longitudinal study of psychological distress in the United States before and during the COVID-19 pandemic. Prev Med 143, 106362 (2021).

11. Hawes, M. T., Szenczy, A. K., Klein, D. N., Hajcak, G. \& Nelson, B. D. Increases in depression and anxiety symptoms in adolescents and young adults during the COVID-19 pandemic. Psychol Med 1-9 (2021) doi:10.1017/S0033291720005358.

12. Meda, N. et al. Students' mental health problems before, during, and after COVID-19 lockdown in Italy. J Psychiatr Res 134, 69-77 (2021).

13. Saraswathi, I. et al. Impact of COVID-19 outbreak on the mental health status of undergraduate medical students in a COVID-19 treating medical college: a 
prospective longitudinal study. PeerJ 8, e10164 (2020).

14. Elmer, T., Mepham, K. \& Stadtfeld, C. Students under lockdown: Comparisons of students' social networks and mental health before and during the COVID-19 crisis in Switzerland. PLOS ONE 15, e0236337 (2020).

15. Li, H. Y., Cao, H., Leung, D. Y. P. \& Mak, Y. W. The Psychological Impacts of a COVID-19 Outbreak on College Students in China: A Longitudinal Study. Int J Environ Res Public Health 17, (2020).

16. Creese, B. et al. Loneliness, physical activity and mental health during Covid-19: a longitudinal analysis of depression and anxiety in adults over 50 between 2015 and 2020. Int Psychogeriatr 1-21 (2020) doi:10.1017/S1041610220004135.

17. Mathews, A. \& MacLeod, C. Cognitive Vulnerability to Emotional Disorders. Annual Review of Clinical Psychology 1, 167-195 (2005).

18. Moffitt, T. E. et al. How common are common mental disorders? Evidence that lifetime prevalence rates are doubled by prospective versus retrospective ascertainment. Psychol Med 40, 899-909 (2010).

19. Dunlop, B. W. et al. Recall accuracy for the symptoms of a major depressive episode among clinical trial participants. Journal of Psychiatric Research 116, 178-184 (2019).

20. Davies, M. R. et al. The Genetic Links to Anxiety and Depression (GLAD) Study: Online recruitment into the largest recontactable study of depression and anxiety. Behav Res Ther 123, 103503 (2019).

21. Kroenke, K., Spitzer, R. L. \& Williams, J. B. The PHQ-9: validity of a brief depression severity measure. J Gen Intern Med 16, 606-613 (2001).

22. Spitzer, R. L., Kroenke, K., Williams, J. B. W. \& Löwe, B. A Brief Measure for Assessing Generalized Anxiety Disorder: The GAD-7. Arch Intern Med 166, 1092 
(2006).

23. Lang, A. J. \& Stein, M. B. An abbreviated PTSD checklist for use as a screening instrument in primary care. Behav Res Ther 43, 585-594 (2005).

24. Why are there discrepancies between depressed patients' Global Rating of Change and scores on the Patient Health Questionnaire depression module? A qualitative study of primary care in England | BMJ Open. https://bmjopen.bmj.com/content/7/4/e014519.

25. Griffiths, C. A. \& Griffiths, L. J. Recovery and reliable change rates for patients scoring severe on depression, anxiety or impaired functioning in a psychological therapies service: IAPT. Mental Health Review Journal 20, 28-35 (2015).

26. Robinson, J. et al. Why are there discrepancies between depressed patients' Global Rating of Change and scores on the Patient Health Questionnaire depression module? A qualitative study of primary care in England. BMJ Open 7, e014519 (2017).

27. Fancourt, D., Steptoe, A. \& Bu, F. Trajectories of anxiety and depressive symptoms during enforced isolation due to COVID-19 in England: a longitudinal observational study. The Lancet Psychiatry 0, (2020).

28. Pierce, M. et al. Says who? The significance of sampling in mental health surveys during COVID-19. The Lancet Psychiatry 7, 567-568 (2020).

29. Barnett, A. G., van der Pols, J. C. \& Dobson, A. J. Regression to the mean: what it is and how to deal with it. International Journal of Epidemiology 34, 215-220 (2005). 


\section{Figure and Table captions}

Figure 1. A) Study design for the COPING and RAMP studies and measures available at each time point. B) Month of study sign-up across cohorts. C) Demographic details of participants across cohorts. ${ }^{1}$ Participants in the NBR group combined EDGI, IBD and general population cohorts. ${ }^{2}$ Not displayed due to scaling, but participant gender also includes Non-binary/Self-defined: GLAD (247), NBR (41), RAMP (92)

Figure 2. Self-reported prior mental health diagnosis by sample (note that participants could report $>1$ diagnosis). GLAD $=$ Genetic Links to Anxiety and Depression cohort $(\mathrm{N}=12,718) ; \mathrm{NBR}=\mathrm{NIHR}$ BioResource cohort $(\mathrm{N}=13,096) ; \mathrm{RAMP}=$ Repeated Assessment of Mental Health in Pandemics cohort $(N=8,651)$. Note that the frequency of mental health diagnoses was approximately double in the RAMP compared to the NBR sample. This suggests that the RAMP online advertising may have particularly appealed to individuals with experience of mental health conditions.

Figure 3. Forest plots detailing the effects of prior mental health diagnosis, gender, age, ethnicity and employment status on current depression, anxiety and PTSD symptoms during the pandemic, controlling for prospectively measured prepandemic symptom levels. Points indicate effect size estimates, error bars represent $95 \%$ confidence intervals. Lower scores indicate a lower burden of symptoms.

Table 1. Sample size, mean and standard deviation of depression (PHQ-9), anxiety (GAD-7) and PTSD (PCL-6) symptoms

\begin{tabular}{ccccccccccc}
\hline & \multicolumn{3}{c}{ Pre-pandemic } & \multicolumn{3}{c|}{ Retrospective } & \multicolumn{3}{c}{ Current (baseline) } \\
\hline & $\mathrm{N}$ & $\mathrm{M}$ & $\mathrm{SD}$ & $\mathrm{N}$ & $\mathrm{M}$ & $\mathrm{SD}$ & $\mathrm{N}$ & $\mathrm{M}$ & $\mathrm{SD}$ \\
\hline Overall & & & & & & & & & & \\
PHQ-9 & - & - & - & 34,223 & 5.39 & 5.71 & 34,386 & 7.79 & 6.91 \\
GAD-7 & - & - & - & 34,334 & 4.14 & 4.67 & 34,405 & 6.11 & 5.94 \\
PCL-6 & - & - & - & - & - & - & 34,323 & 12.46 & 6.04 \\
GLAD & & & & & & & & & \\
PHQ-9 & 12,098 & 11.18 & 6.86 & 12,562 & 8.07 & 6.16 & 12,608 & 10.80 & 6.68 \\
GAD-7 & 12,108 & 8.78 & 5.96 & 12,597 & 6.14 & 5.01 & 12,613 & 8.48 & 5.83 \\
PCL-6 & 12,030 & 15.40 & 6.09 & - & - & - & 12,547 & 15.71 & 6.16 \\
NBR & & & & & & & & & \\
PHQ-9 & - & - & - & 13,087 & 2.20 & 3.25 & 13,147 & 3.31 & 4.13 \\
GAD-7 & - & - & - & 13,137 & 1.65 & 2.81 & 13,156 & 2.44 & 3.66 \\
PCL-6 & - & - & - & - & - & - & 13,161 & 8.54 & 3.51 \\
RAMP & & & & & & & & & \\
PHQ-9 & - & - & - & 8,574 & 6.20 & 5.70 & 8,631 & 10.24 & 7.02 \\
GAD-7 & - & - & - & 8,600 & 5.02 & 4.71 & 8,636 & 8.26 & 6.12 \\
PCL-6 & - & - & - & - & - & - & 8,615 & 13.72 & 5.54 \\
\hline
\end{tabular}




\section{A) Study design}

\begin{tabular}{|c|c|c|}
\hline \multicolumn{2}{|c|}{ COPING } & \multirow{2}{*}{ RAMP } \\
\hline GLAD & NBR $^{1}$ & \\
\hline \multicolumn{3}{|l|}{$\begin{array}{l}\text { Pre-pandemic } \\
\text { PHQ-9, GAD-7, PCL-6 }\end{array}$} \\
\hline $\begin{array}{l}\text { Retrospective } \\
\text { PHQ-9, GAD-7 }\end{array}$ & $\begin{array}{l}\text { Retrospective } \\
\text { PHQ-9, GAD-7 }\end{array}$ & $\begin{array}{l}\text { Retrospective } \\
\text { PHQ-9, GAD-7 }\end{array}$ \\
\hline $\begin{array}{c}\text { Current } \\
\text { Demographics, PHQ-9 (+ GRC), } \\
\text { GAD-7 (+ GRC), PCL-6 }\end{array}$ & $\begin{array}{c}\text { Current } \\
\text { Demographics, PHQ-9 (+ GRC), } \\
\text { GAD-7 (+ GRC), PCL-6 }\end{array}$ & $\begin{array}{c}\text { Current } \\
\text { Demographics, PHQ-9 (+ GRC), } \\
\text { GAD-7 (+ GRC), PCL-6 }\end{array}$ \\
\hline
\end{tabular}

\section{B) Study sign-up}

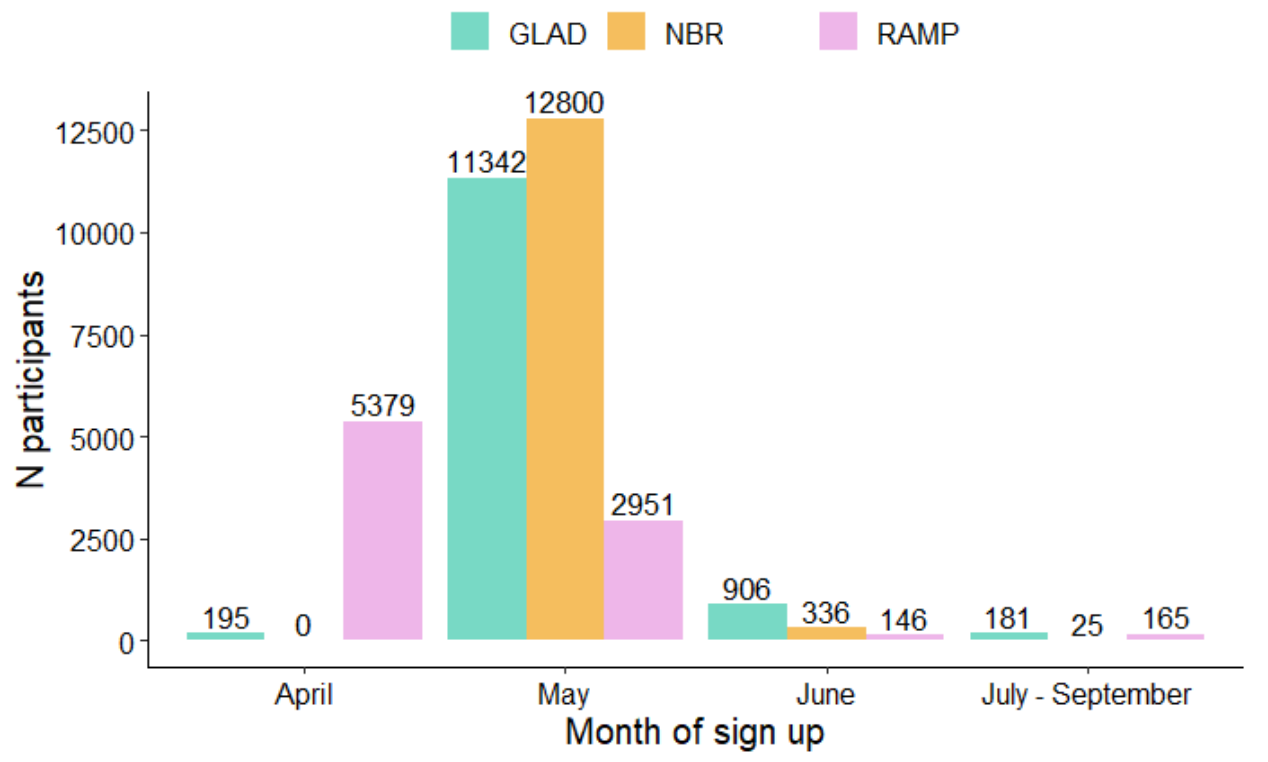

\section{C) Cohort demographics}
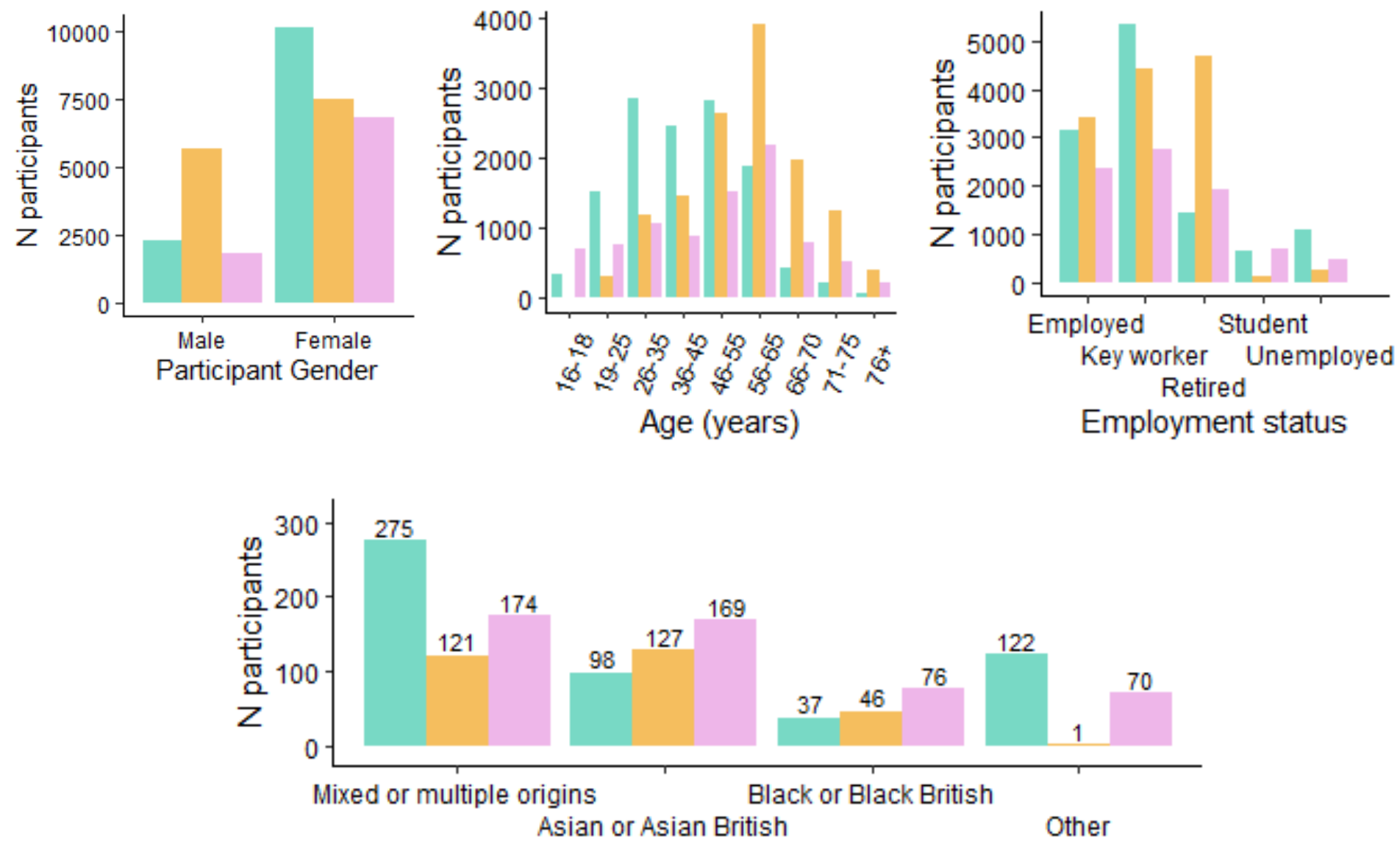


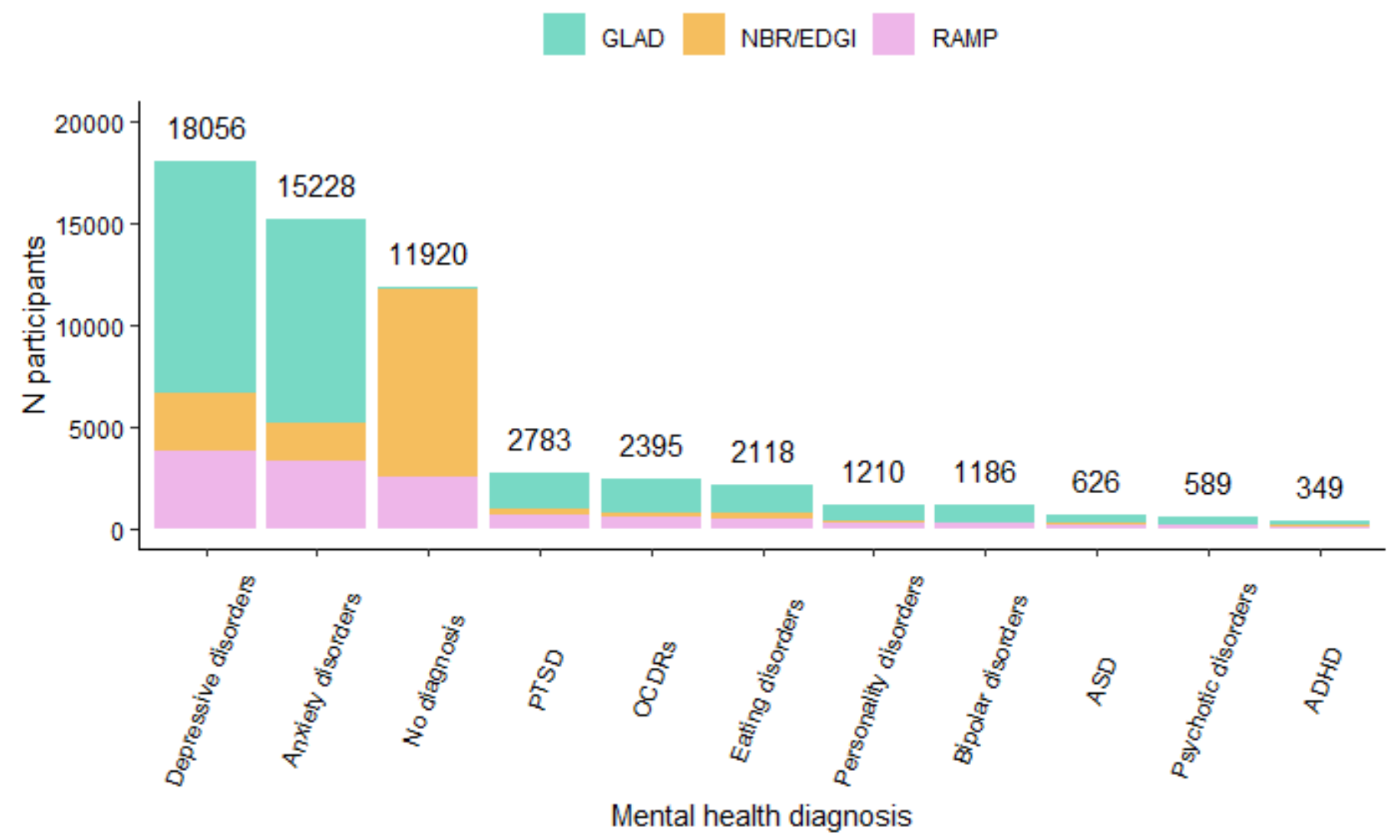

Figure 2 
PHQ total prepandemic

PCL total prepandemic

GAD total prepandemic

Comorbid depression and anxiety

Depressive disorders

Anxiety disorders

Eating disorders

Obsessive compulsive disorders

Comorbid psychotic and bipolar disorders

Psychotic disorders

Bipolar disorder

Post traumatic stress disorder

Autism spectrum disorder

Attention deficit hyperactivity disorder

Personality disorders

$-2$
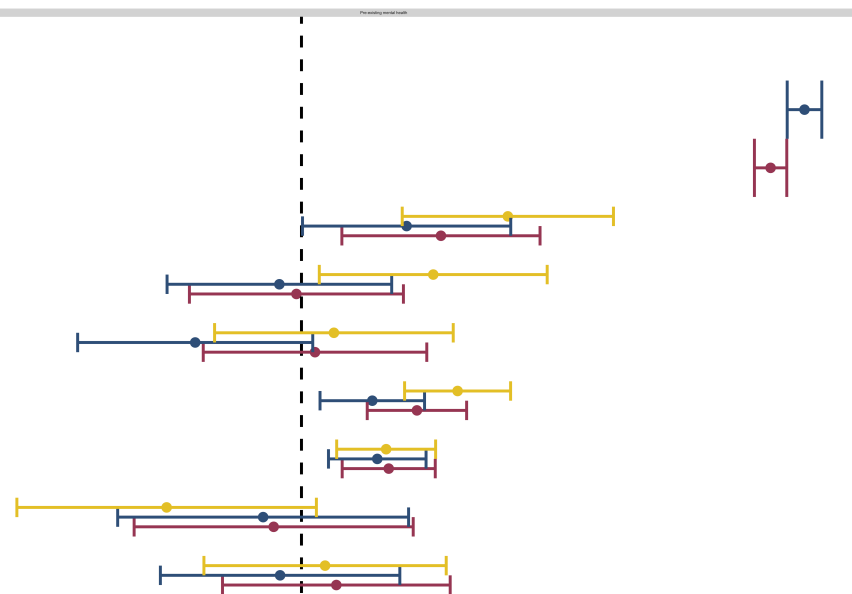

Female

Non-binary/Self-defined

$-2$

1 上曰=-

$\stackrel{1}{1}$

2

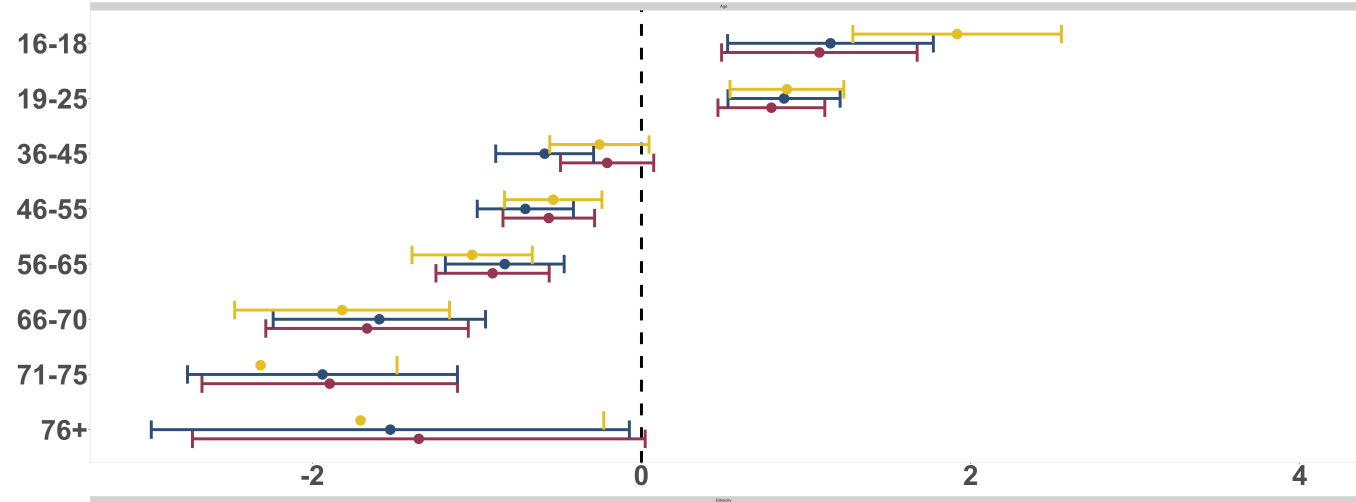

Mixed or multiple ethnicities

Asian or Asian British

Black or Black British

Other

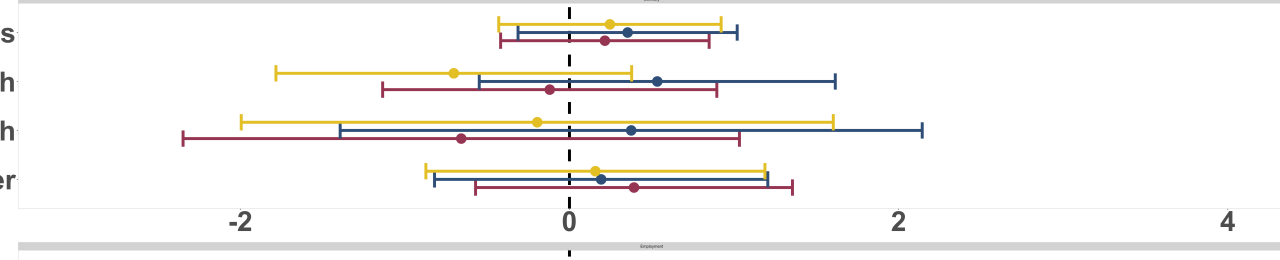

Key worker

Retired

Student

Unemployed

Time

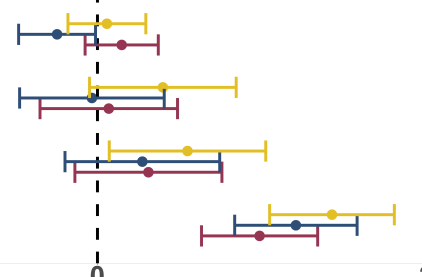

$-2$

0

2

4

$-2$

0

2

4

Estimate (95\% Confidence Interval)

-PHQ-9 •PCL-6 • GAD-7

Figure 3 


\section{Supplementary materials}

\section{Assessment of mental health diagnoses}

History of prior mental health diagnoses was assessed using a checklist (Table S1) following the prompt: "Have you ever been diagnosed with one or more of the following mental health problems by a professional, even if you don't have it currently? By professional we mean: any doctor, nurse or person with specialist training (such as a psychologist, psychiatrist etc.). Please include disorders even if you did not need treatment for them or if you did not agree with the diagnosis."

For two sets of diagnoses, we created separate 'comorbid' and 'single diagnosis' category variables due to high levels of correlations between diagnostic categories. These groups were depressive disorders and anxiety disorders $(r=0.84)$ and psychotic disorders and bipolar disorders $(r=0.71)$. For regression analyses, these categories were re-coded as: i) 'comorbid depressive and anxiety disorder', 'only depressive disorder' and 'only anxiety disorder; ii) 'comorbid psychotic and bipolar disorder', 'only psychotic disorder' and 'only bipolar disorder'.

Table S1. Diagnosis questionnaire response options and categorisation used in analyses

\begin{tabular}{|c|c|}
\hline Individual diagnoses (response options) & Category \\
\hline Depression & Depressive disorder only \\
\hline Depression during or after pregnancy (antenatal/postnatal depression) & Depressive disorder only \\
\hline Premenstrual dysphoric disorder (PMDD) & Depressive disorder only \\
\hline Anxiety, nerves or generalised anxiety disorder & Anxiety disorder only \\
\hline Social anxiety or social phobia & Anxiety disorder only \\
\hline Specific phobia (e.g. phobia of flying) & Anxiety disorder only \\
\hline Agoraphobia & Anxiety disorder only \\
\hline Panic disorder & Anxiety disorder only \\
\hline Panic attacks & Anxiety disorder only \\
\hline $\begin{array}{l}\text { NOTE: If both depression and anxiety were present, a separate category of } \\
\text { comorbid depressive and anxiety disorder was created }\end{array}$ & Depressive and anxiety disorder \\
\hline Post-traumatic stress disorder (PTSD) & PTSD \\
\hline Obsessive-compulsive disorder (OCD) & OCRDs \\
\hline Body dysmorphic disorder (BDD) & OCRDs \\
\hline Other obsessive-compulsive related disorder e.g. skin picking & OCRDs \\
\hline Anorexia nervosa & Eating disorders \\
\hline Atypical anorexia nervosa & Eating disorders \\
\hline Bulimia nervosa & Eating disorders \\
\hline Psychological overeating or binge-eating disorder & Eating disorders \\
\hline Mania, hypomania, bipolar or manic-depression & Bipolar disorder \\
\hline Schizophrenia & Psychosis only \\
\hline Schizoaffective disorder & Psychosis only \\
\hline Any other type of psychosis or psychotic illness & Psychosis only \\
\hline $\begin{array}{l}\text { NOTE: If both bipolar and psychosis were present, a separate category of } \\
\text { comorbid psychotic and bipolar disorder was created }\end{array}$ & Psychotic and bipolar disorder \\
\hline Personality disorder & Personality disorder \\
\hline Autism, asperger's or autistic spectrum disorder & ASD \\
\hline Attention deficit or attention deficit and hyperactivity disorder (ADD/ADHD) & ADHD \\
\hline
\end{tabular}

\section{Details of symptom measures}

Patient Health Questionnaire - 9 items (PHQ-9)

The PHQ-9 is a 9-item self-report measure of DSM-IV diagnostic criteria for major depressive disorder (MDD). Each item is a diagnostic symptom of MDD and is rated on a 0 (not at all) to 3 (nearly every day) scale, producing a range of scores from 0 to 27 . The PHQ-9 has a test-retest reliability of 0.84 and a cut-off score of $\geq 10$ has a sensitivity of $88 \%$, a specificity of $88 \%$ for major depression ${ }^{1}$. 
Generalized anxiety disorder scale - 7 items (GAD-7)

The GAD-7 is a 7-item self-report screening and severity measure for generalised anxiety disorder (GAD). Each item is rated on a 0 (not at all) to 3 (nearly every day) scale, producing a range of scores from 0 to 21 . The GAD-7 has a test-retest reliability of 0.83 , and a cut-off score of $\geq 10$ has a sensitivity of $89 \%$ and a specificity of $82 \%$ for $\mathrm{GAD}^{2}$.

Abbreviated PTSD Checklist - 6 items (PCL-6)

The PCL-6 is a 6-item self-report screening and severity measure of PTSD symptoms. Each item is rated on a 1 (not at all) to 5 (extremely) scale, producing a range of scores from 6 to 30 . A cut-off score of $\geq 14$ has a sensitivity of $80 \%$, a specificity of $76 \%$ for PTSD. In the current study, the third item of the PCL-6 ('Avoiding activities or situations because they reminded you of a stressful experience from the past') was adapted for current (during the pandemic) symptom assessment by adding 'this does not include activities or situations that are currently restricted or advised against'.

Global rating of change and retrospective ratings

After completing current symptom measures (PHQ-9 and GAD-7 only), participants provided a global rating of change, assessing perceived changes in symptoms ${ }^{3}$ ('How different are these feelings to how you felt before the pandemic?'), on a 5-point scale ('much worse', to 'much better'). Indication of symptom change was followed up with retrospective assessment of PHQ-9 and GAD-7 to estimate pre-pandemic symptoms (prompt: 'thinking about how you usually felt before the pandemic...'). Retrospective scores were imputed from current scores for participants rating 'no difference' in symptoms. 
Table S2. Demographic details of participants across cohorts. Percentages denote the proportion of individuals within a given category for each cohort (GLAD, NBR or RAMP) or in the combined sample (overall)

\begin{tabular}{|c|c|c|c|c|c|c|c|c|}
\hline & \multicolumn{2}{|c|}{ GLAD $^{1}$} & \multicolumn{2}{|c|}{ NBR } & \multicolumn{2}{|c|}{ RAMP } & \multicolumn{2}{|c|}{ Overall } \\
\hline & $\mathbf{N}$ & $\%$ & $\mathbf{N}$ & $\%$ & $\mathbf{N}$ & $\%$ & $\mathbf{N}$ & $\%$ \\
\hline$\overline{\text { Total N }}$ & 12,653 & & 13,161 & & 8,651 & & 34,465 & \\
\hline \multicolumn{9}{|l|}{ Psychiatric diagnosis } \\
\hline Depressive and anxiety disorder & 9236 & $72.8 \%$ & 1119 & $8.5 \%$ & 2551 & $29.5 \%$ & 12906 & $37.5 \%$ \\
\hline Only depressive disorder & 1359 & $10.7 \%$ & 1637 & $12.5 \%$ & 1272 & $14.7 \%$ & 4268 & $12.4 \%$ \\
\hline Only anxiety disorder & 840 & $6.6 \%$ & 646 & $4.9 \%$ & 798 & $9.2 \%$ & 2284 & $6.6 \%$ \\
\hline PTSD & 1846 & $14.5 \%$ & 237 & $1.8 \%$ & 700 & $8.1 \%$ & 2783 & $8.1 \%$ \\
\hline OCRDs & 1625 & $12.8 \%$ & 163 & $1.2 \%$ & 607 & $7.0 \%$ & 2395 & $7.0 \%$ \\
\hline Eating disorders & 1380 & $10.9 \%$ & 256 & $2.0 \%$ & 482 & $5.6 \%$ & 2118 & $6.2 \%$ \\
\hline Personality disorders & 950 & $7.5 \%$ & $82^{3}$ & $0.6 \%$ & 295 & $3.4 \%$ & 1327 & $3.9 \%$ \\
\hline Psychotic and bipolar disorder & 168 & $1.3 \%$ & $8^{3}$ & $0.1 \%$ & $76^{3}$ & $0.9 \%$ & 252 & $0.7 \%$ \\
\hline Only psychotic disorders & 232 & $1.8 \%$ & $29^{3}$ & $0.2 \%$ & $76^{3}$ & $0.9 \%$ & 337 & $1.0 \%$ \\
\hline Only bipolar disorders & 701 & $5.5 \%$ & $67^{3}$ & $0.5 \%$ & 148 & $1.7 \%$ & 916 & $2.7 \%$ \\
\hline ASD & 383 & $3.0 \%$ & $52^{3}$ & $0.4 \%$ & 191 & $2.2 \%$ & 626 & $1.8 \%$ \\
\hline ADHD & 201 & $1.6 \%$ & $34^{3}$ & $0.3 \%$ & 114 & $1.3 \%$ & 349 & $1.0 \%$ \\
\hline No diagnosis & 137 & $1.1 \%$ & 9248 & $70.6 \%$ & 2535 & $29.3 \%$ & 11920 & $34.6 \%$ \\
\hline \multicolumn{9}{|l|}{ Gender } \\
\hline Male & 2,271 & 17.9 & 5,642 & 42.9 & 1,771 & 20.5 & 9,684 & 28.1 \\
\hline Female & 10,106 & 79.9 & 7,478 & 59.1 & 6,778 & 78.3 & 24,362 & 70.7 \\
\hline Non-binary/self-defined & 247 & 2.0 & $41^{3}$ & 0.3 & $92^{3}$ & 1.1 & 380 & 1.1 \\
\hline \multicolumn{9}{|l|}{ Age } \\
\hline $16-18$ & 340 & 2.7 & $17^{3}$ & 0.1 & 697 & 8.1 & 1,054 & 3.1 \\
\hline $19-25$ & 1,537 & 12.1 & 308 & 2.3 & 772 & 8.9 & 2,617 & 7.6 \\
\hline $26-35$ & 2,850 & 22.5 & 1,186 & 9.0 & 1,076 & 12.4 & 5,112 & 14.8 \\
\hline $36-45$ & 2,453 & 19.4 & 1,448 & 11.0 & 874 & 10.1 & 4,775 & 13.9 \\
\hline $46-55$ & 2,814 & 22.2 & 2,648 & 20.2 & 1,518 & 17.5 & 6,980 & 20.3 \\
\hline $56-65$ & 1,900 & 15.0 & 3,906 & 29.7 & 2,200 & 25.4 & 8,006 & 23.2 \\
\hline $66-70$ & 428 & 3.4 & 1,970 & 15.0 & 784 & 9.1 & 3,182 & 9.2 \\
\hline $71-75$ & 230 & 1.8 & 1,265 & 9.6 & 511 & 5.9 & 2,006 & 5.8 \\
\hline $76+$ & $72^{3}$ & 0.6 & 413 & 3.1 & 209 & 2.4 & 694 & 2.0 \\
\hline \multicolumn{9}{|l|}{ Ethnicity $^{2}$} \\
\hline White & 12,062 & 95.3 & 9,987 & 75.9 & 8,114 & 93.8 & 30,163 & 87.5 \\
\hline Asian or Asian British & $98^{3}$ & 0.8 & 127 & 1.0 & 169 & 2.0 & 394 & 1.1 \\
\hline Black or Black British & $37^{3}$ & 0.3 & $46^{3}$ & 0.3 & $76^{3}$ & 0.9 & 159 & 0.5 \\
\hline Mixed or multiple ethnic origins & 275 & 2.2 & 121 & 0.9 & 174 & 2.0 & 570 & 1.7 \\
\hline Other ethnicity & $30^{3}$ & 1.0 & $1^{3}$ & 0.0 & $70^{3}$ & 0.8 & 193 & 0.6 \\
\hline \multicolumn{9}{|l|}{ Employment } \\
\hline Employed & 3,157 & 25.0 & 3,414 & 25.9 & 2,345 & 27.1 & 8,916 & 25.9 \\
\hline Employed as key worker & 5,346 & 42.3 & 4,437 & 33.7 & 2,763 & 31.9 & 12,546 & 36.4 \\
\hline Retired & 1,427 & 11.3 & 4,681 & 35.5 & 1,908 & 22.1 & 8,021 & 23.3 \\
\hline Student & 672 & 5.3 & 114 & 0.9 & 720 & 8.3 & 1,506 & 4.4 \\
\hline Unemployed & 1,103 & 8.7 & 250 & 1.9 & 500 & 5.8 & 1,853 & 5.4 \\
\hline
\end{tabular}

Note: GLAD and NBR combined form the COPING study, ${ }^{1}$ pre-pandemic mental health data available, ${ }^{2}$ where available, ${ }^{3}$ note that in individual cohort analyses, these factors do not meet a priori power calculation criteria 


\section{Statistical analysis}

Power calculation

We conducted a priori power calculations using $\mathrm{G}^{*}$ power for $\mathrm{t}$-tests (mean difference between 2 independent groups), $80 \%$ power, alpha $0.05, d=0.3$. Even group size required $n=176$ per group, uneven group size (ratio 1:6) required $n=102$ for the smaller group. Thus, in our analyses of combined samples presented here, there is sufficient power to examine effects of ethnicity. For consistency across models, we include ethnicity in all analyses presented, noting that for individual samples the threshold group size is not met for ethnicity.

Variable coding details

Individual diagnoses were combined to create the following categories: i) depression and anxiety, ii) depression only, iii) anxiety only, iv) eating disorders, v) obsessive compulsive disorders, vi) psychotic and bipolar disorders, vii) psychotic disorder only, viii) bipolar disorder only, ix) post-traumatic stress disorder, $\mathrm{x}$ ) autism spectrum disorders, $x i)$ attention deficit hyperactivity disorder, xii) personality disorder .All demographic factors were binary coded. Age was coded categorically with binary yes/no coding in each age group. The reference categories for demographic variables in regression analyses were as follows: gender $=$ male; age $=26-35$ years (the largest age group); ethnicity $=$ White; employment $=$ employed.

\section{Results: Symptom change}

Table S3. Reported changes in symptoms of depression (PHQ-9) and anxiety (GAD-7) by sample (GLAD, NBR, RAMP)

\begin{tabular}{llrrrrr}
\hline Sample & Measure & \multicolumn{5}{c}{ Perceived change (\% of sample) } \\
\hline & & Much worse & A little worse & No Change & A little better & Much better \\
\hline GLAD & PHQ-9 & $18.1 \%$ & $44.7 \%$ & $25.3 \%$ & $8.2 \%$ & $3.5 \%$ \\
& GAD-7 & $19.7 \%$ & $42.3 \%$ & $28.4 \%$ & $6.8 \%$ & $3.2 \%$ \\
\multirow{2}{*}{ RBR } & PHQ-9 & $6.9 \%$ & $33.0 \%$ & $56.0 \%$ & $2.6 \%$ & $1.0 \%$ \\
& GAD-7 & $6.8 \%$ & $29.7 \%$ & $60.4 \%$ & $2.6 \%$ & $0.9 \%$ \\
\multirow{2}{*}{ Overall } & PHQ-9 & $26.6 \%$ & $43.8 \%$ & $21.8 \%$ & $4.9 \%$ & $2.7 \%$ \\
& GAD-7 & $27.4 \%$ & $41.5 \%$ & $24.7 \%$ & $4.2 \%$ & $2.3 \%$ \\
& PHQ-9 & $15.9 \%$ & $40.0 \%$ & $36.2 \%$ & $5.2 \%$ & $2.4 \%$ \\
& GAD-7 & $16.7 \%$ & $37.3 \%$ & $39.7 \%$ & $4.5 \%$ & $2.1 \%$ \\
\hline
\end{tabular}




\section{Results: Individual differences - regression models}

Table S5. Individual differences in prospectively measured symptom change (GLAD sample). Estimates, and confidence intervals of pre-existing diagnostic and demographic variables (statistically significant effects are in bold).

\begin{tabular}{|c|c|c|c|}
\hline & Depression (PHQ-9) & Anxiety (GAD-7) & PTSD (PCL-6) \\
\hline Intercept & $2.51[1.66,3.37]$ & $3.00[2.20,3.80]$ & $6.29[5.43,7.15]$ \\
\hline Pre-pandemic symptoms * & $0.54[0.53,0.56]$ & $0.48[0.46,0.49]$ & $0.50[0.48,0.52]$ \\
\hline \multicolumn{4}{|l|}{ Psychiatric diagnoses } \\
\hline Depressive and anxiety disorder & $1.25[0.61,1.90]$ & $0.85[0.25,1.45]$ & $0.64[0.01,1.27]$ \\
\hline Only depressive disorder & $0.80[0.11,1.49]$ & $-0.03[-0.68,0.62]$ & $-0.13[-0.82,0.55]$ \\
\hline Only anxiety disorder & $0.20[-0.53,0.92]$ & $0.08[-0.60,0.76]$ & $-0.65[-1.36,0.07]$ \\
\hline Eating disorders & $0.95[0.63,1.27]$ & $0.70[0.40,1.00]$ & $0.43[0.11,0.75]$ \\
\hline OCRDs & $0.51[0.21,0.82]$ & $0.53[0.25,0.81]$ & $0.46[0.16,0.76]$ \\
\hline Psychotic and bipolar disorder & $-0.82[-1.73,0.09]$ & $-0.17[-1.02,0.68]$ & $-0.23[-1.12,0.65]$ \\
\hline Only psychotic disorder & $0.14[-0.59,0.88]$ & $0.21[-0.48,0.90]$ & $-0.13[-0.86,0.60]$ \\
\hline Only bipolar disorder & $0.27[-0.17,0.72]$ & $0.24[-0.17,0.66]$ & $0.44[0.00,0.88]$ \\
\hline PTSD & $0.57[0.28,0.86]$ & $0.78[0.51,1.04]$ & $0.80[0.51,1.09]$ \\
\hline ASD & $0.66[0.06,1.25]$ & $0.74[0.18,1.30]$ & $0.45[-0.14,1.04]$ \\
\hline ADHD & $0.35[-0.47,1.16]$ & $-0.24[-1.01,0.52]$ & $0.34[-0.48,1.16]$ \\
\hline Personality disorder & $0.90[0.49,1.31]$ & $0.58[0.19,0.97]$ & $0.65[0.25,1.06]$ \\
\hline \multicolumn{4}{|l|}{ Gender } \\
\hline Female & $0.15[-0.12,0.41]$ & $0.40[0.15,0.64]$ & $0.52[0.25,0.78]$ \\
\hline Non-binary/Self-defined & $0.28[-0.48,1.03]$ & $0.44[-0.27,1.14]$ & $0.52[-0.22,1.27]$ \\
\hline \multicolumn{4}{|l|}{ Age } \\
\hline 16-18 years & $1.92[1.28,2.55]$ & $1.08[0.49,1.67]$ & $1.15[0.52,1.77]$ \\
\hline $19-25$ years & $0.88[0.54,1.23]$ & $0.79[0.46,1.11]$ & $0.87[0.53,1.21]$ \\
\hline $36-45$ years & $-0.26[-0.56,0.05]$ & $-0.21[-0.49,0.07]$ & $-0.59[-0.89,-0.29]$ \\
\hline $46-55$ years & $-0.54[-0.83,-0.24]$ & $-0.56[-0.84,-0.28]$ & $-0.71[-1.00,-0.41]$ \\
\hline $56-65$ years & $-1.03[-1.39,-0.66]$ & $-0.90[-1.25,-0.56]$ & $-0.83[-1.19,-0.47]$ \\
\hline $66-70$ years & $-1.82[-2.47,-1.17]$ & $-1.67[-2.28,-1.05]$ & $-1.59[-2.24,-0.95]$ \\
\hline $71-75$ years & $-2.31[-3.14,-1.48]$ & $-1.89[-2.67,-1.12]$ & $-1.94[-2.76,-1.12]$ \\
\hline $76+$ years & $-1.71[-3.18,-0.23]^{\$}$ & $-1.35[-2.73,0.02]^{\$}$ & $-1.53[-2.98,-0.07]$ \\
\hline \multicolumn{4}{|l|}{ Ethnicity } \\
\hline Asian or Asian British & $-0.70[-1.78,0.38]^{\$}$ & $-0.12[-1.14,0.90]^{\$}$ & $0.53[-0.55,1.62]^{\$}$ \\
\hline Black or Black British & $-0.20[-1.99,1.60]^{\$}$ & $-0.66[-2.35,1.03]^{\$}$ & $0.37[-1.39,2.14]^{\$}$ \\
\hline Mixed or multiple ethnic origins & $0.25[-0.43,0.92]$ & $0.22[-0.42,0.85]$ & $0.35[-0.31,1.02]$ \\
\hline Other ethnicity & $0.16[-0.87,1.19]$ & $0.39[-0.57,1.35]$ & $0.19[-0.82,1.21]$ \\
\hline \multicolumn{4}{|l|}{ Employment } \\
\hline Key worker & $0.06[-0.18,0.29]$ & $0.15[-0.08,0.37]$ & $-0.25[-0.48,-0.01]$ \\
\hline Retired & $0.40[-0.05,0.84]$ & $0.07[-0.35,0.49]$ & $-0.03[-0.47,0.41]$ \\
\hline Student & $0.55[0.07,1.02]$ & $0.31[-0.14,0.76]$ & $0.27[-0.20,0.74]$ \\
\hline Unemployed & $1.42[1.05,1.80]$ & $0.98[0.63,1.34]$ & $1.21[0.83,1.58]$ \\
\hline \multicolumn{4}{|l|}{ Time of completion } \\
\hline Survey completion date & $0.08[0.01,0.14]$ & $0.00[-0.06,0.05]$ & $0.10[0.04,0.16]$ \\
\hline
\end{tabular}

* pre-pandemic symptoms varied across models - PHQ-9 scores were entered for depression model, GAD-7 scores were entered for anxiety model and PCL-6 scores were entered for PTSD model, ${ }^{\$}$ sample sizes for these effects did not meet a priori power criteria (based on combined sample) so effects should be interpreted with caution 
Table S6. Aim 2b: Individual differences in retrospectively estimated symptom change (combined sample). Estimates, confidence intervals and significance levels of pre-existing diagnostic and demographic variables (statistically significant effects are in bold).

\begin{tabular}{|c|c|c|}
\hline & Depression (PHQ-9) & Anxiety (GAD-7) \\
\hline Intercept & $4.10[3.84,4.37]$ & $3.64[3.39,3.88]$ \\
\hline Pre-pandemic symptoms * & $0.68[0.66,0.69]$ & $0.66[0.65,0.67]$ \\
\hline \multicolumn{3}{|l|}{ Psychiatric diagnoses } \\
\hline Depressive and anxiety disorder & $2.68[2.53,2.83]$ & $2.41[2.27,2.54]$ \\
\hline Only depressive disorder & $1.69[1.52,1.86]$ & $1.02[0.86,1.18]$ \\
\hline Only anxiety disorder & $1.18[0.96,1.40]$ & $1.76[1.56,1.97]$ \\
\hline Eating disorders & $0.79[0.56,1.01]$ & $0.55[0.34,0.76]$ \\
\hline OCRDs & $0.46[0.24,0.67]$ & $0.47[0.27,0.68]$ \\
\hline Psychotic and bipolar disorder & $0.02[-0.61,0.65]$ & $0.72[0.14,1.30]$ \\
\hline Only psychotic disorder & $-0.05[-0.59,0.49]$ & $0.00[-0.50,0.50]$ \\
\hline Only bipolar disorder & $0.64[0.30,0.99]$ & $0.32[0.00,0.64]$ \\
\hline PTSD & $0.93[0.73,1.13]$ & $0.77[0.58,0.96]$ \\
\hline ASD & $0.55[0.15,0.95]$ & $0.65[0.28,1.02]$ \\
\hline ADHD & $0.34[-0.19,0.86]$ & $0.10[-0.39,0.58]$ \\
\hline Personality disorder & $0.80[0.50,1.10]$ & $0.44[0.17,0.72]$ \\
\hline \multicolumn{3}{|l|}{ Gender } \\
\hline Female & $0.74[0.61,0.86]$ & $0.78[0.66,0.89]$ \\
\hline Non-binary/Self-defined & $0.54[0.03,1.05]$ & $0.13[-0.34,0.60]$ \\
\hline \multicolumn{3}{|l|}{ Age } \\
\hline $16-18$ years & $2.82[2.46,3.17]$ & $0.89[0.56,1.21]$ \\
\hline $19-25$ years & $1.20[0.96,1.43]$ & $0.89[0.67,1.11]$ \\
\hline $36-45$ years & $-0.59[-0.79,-0.40]$ & $-0.57[-0.75,-0.39]$ \\
\hline $46-55$ years & $-0.77[-0.95,-0.59]$ & $-0.87[-1.04,-0.70]$ \\
\hline $56-65$ years & $-1.16[-1.35,-0.97]$ & $-1.19[-1.37,-1.01]$ \\
\hline $66-70$ years & $-1.59[-1.86,-1.32]$ & $-1.58[-1.83,-1.33]$ \\
\hline $71-75$ years & $-1.75[-2.05,-1.45]$ & $-1.78[-2.06,-1.50]$ \\
\hline $76+$ years & $-1.77[-2.19,-1.35]$ & $-1.82[-2.20,-1.43]$ \\
\hline \multicolumn{3}{|l|}{ Ethnicity } \\
\hline Asian or Asian British & $0.00[-0.47,0.47]$ & $-0.19[-0.62,0.25]$ \\
\hline Black or Black British & $-0.43[-1.32,0.46]$ & $-0.65[-1.48,0.18]$ \\
\hline Mixed or multiple ethnic origins & $0.12[-0.28,0.52]$ & $-0.19[-0.56,0.18]$ \\
\hline Other ethnicity & $0.48[-0.24,1.20]$ & $0.40[-0.27,1.07]$ \\
\hline \multicolumn{3}{|l|}{ Employment } \\
\hline Key worker & $0.11[-0.02,0.24]$ & $0.19[0.06,0.31]$ \\
\hline Retired & $-0.19[-0.39,0.00]$ & $-0.12[-0.30,0.06]$ \\
\hline Student & $0.44[0.15,0.74]$ & $0.34[0.07,0.61]$ \\
\hline Unemployed & $0.65[0.40,0.90]$ & $0.42[0.19,0.64]$ \\
\hline \multicolumn{3}{|l|}{ Time of completion } \\
\hline Survey completion date & $-0.22[-0.25,-0.19]$ & $-0.22[-0.24,-0.19]$ \\
\hline
\end{tabular}

* prepandemic symptoms were different for each model - PHQ-9 scores were entered for depression model,

GAD-7 scores were entered for anxiety model 


\section{Results: individual cohort regression analyses}

Overall, the pattern of effects observed in analyses for each cohort individually were comparable to that observed in the combined sample. Specific differences observed are noted below.

Comparing the combined model to the individual sample models in relation to depression symptoms, there was no significant effect of: i) only anxiety disorder diagnosis in GLAD and NBR; ii) OCRDs in NBR or RAMP; iii) only bipolar disorder in GLAD and NBR; iv) ASD diagnosis in any of the individual samples (this diagnosis was significant in the combined model); v) personality disorder diagnosis in NBR. In relation to demographic variables, there was no significant effect of female gender in GLAD, non-binary/self-defined gender in GLAD or NBR, and student or unemployment status in NBR or RAMP.

Table S7. Individual differences in retrospectively estimated PHQ-9 symptom change. Estimates, confidence intervals and significance levels of pre-existing diagnostic and demographic variables (statistically significant effects are in bold).

\begin{tabular}{|c|c|c|c|}
\hline & GLAD & NBR & RAMP \\
\hline Intercept & $5.11[4.36,5.85]$ & $1.87[1.67,2.08]$ & $4.94[4.42,5.45]$ \\
\hline Pre-pandemic PHQ-9 & $0.61[0.59,0.62]$ & $0.87[0.85,0.89]$ & $0.61[0.58,0.63]$ \\
\hline \multicolumn{4}{|l|}{ Psychiatric diagnoses } \\
\hline Depressive and anxiety disorder & $0.89[0.23,1.56]$ & $1.15[0.95,1.35]$ & $2.78[2.45,3.11]$ \\
\hline Only depressive disorder & $0.64[-0.07,1.36]$ & $0.65[0.50,0.79]$ & $1.19[0.83,1.56]$ \\
\hline Only anxiety disorder & $-0.46[-1.21,0.29]$ & $0.20[-0.02,0.42]$ & $0.84[0.43,1.26]$ \\
\hline Eating disorders & $0.91[0.58,1.24]$ & $0.68[0.33,1.03]$ & $0.80[0.28,1.32]$ \\
\hline OCRDs & $0.43[0.12,0.73]$ & $0.08[-0.37,0.53]$ & $0.43[-0.08,0.93]$ \\
\hline Psychotic and bipolar disorder & $-0.50[-1.42,0.42]$ & $-1.70[-3.40,0.00]$ & $0.01[-1.28,1.29]$ \\
\hline Only psychotic disorder & $0.10[-0.66,0.87]$ & $0.49[-0.49,1.46]^{\$}$ & $-0.84[-2.14,0.45]$ \\
\hline Only bipolar disorder & $0.40[-0.06,0.86]$ & $0.36[-0.36,1.09]^{\$}$ & $1.16[0.23,2.09]$ \\
\hline PTSD & $0.75[0.46,1.04]$ & $0.83[0.45,1.21]$ & $0.89[0.43,1.35]$ \\
\hline ASD & $0.44[-0.17,1.05]$ & $0.10[-0.65,0.84]^{\$}$ & $0.77[-0.03,1.56]$ \\
\hline ADHD & $0.54[-0.30,1.38]$ & $-0.77[-1.70,0.16]^{\$}$ & $-0.10[-1.08,0.88]$ \\
\hline Personality disorder & $0.95[0.54,1.37]$ & $0.22[-0.45,0.89]^{\$}$ & $1.02[0.30,1.75]$ \\
\hline \multicolumn{4}{|l|}{ Gender } \\
\hline Female & $0.15[-0.13,0.43]$ & $0.40[0.30,0.50]$ & $0.99[0.66,1.31]$ \\
\hline Non-binary/Self-defined & $-0.19[-0.95,0.56]$ & $0.35[-0.49,1.18]^{\$}$ & $1.90[0.69,3.11]^{\S}$ \\
\hline \multicolumn{4}{|l|}{ Age } \\
\hline $16-18$ years & $1.91[1.28,2.53]$ & $3.13[1.63,4.63]^{\$}$ & $2.42[1.79,3.05]$ \\
\hline $19-25$ years & $1.05[0.70,1.39]$ & $0.81[0.40,1.22]$ & $1.35[0.81,1.88]$ \\
\hline $36-45$ years & $-0.45[-0.76,-0.14]$ & $-0.63[-0.85,-0.41]$ & $-0.57[-1.08,-0.05]$ \\
\hline $46-55$ years & $-0.47[-0.78,-0.16]$ & $-0.88[-1.08,-0.68]$ & $-0.85[-1.31,-0.40]$ \\
\hline $56-65$ years & $-1.20[-1.59,-0.82]$ & $-1.21[-1.41,-1.01]$ & $-0.98[-1.42,-0.53]$ \\
\hline $66-70$ years & $-1.66[-2.39,-0.93]$ & $-1.45[-1.69,-1.21]$ & $-1.40[-2.01,-0.79]$ \\
\hline $71-75$ years & $-2.48[-3.42,-1.54]$ & $-1.56[-1.81,-1.30]$ & $-1.53[-2.22,-0.84]$ \\
\hline $76+$ years & $-2.34[-3.93,-0.76]^{\$}$ & $-1.57[-1.90,-1.24]$ & $-2.25[-3.16,-1.34]$ \\
\hline \multicolumn{4}{|l|}{ Ethnicity } \\
\hline Asian or Asian British & $-0.31[-1.42,0.79]^{\$}$ & $0.06[-0.37,0.49]$ & $-0.37[-1.25,0.51]$ \\
\hline Black or Black British & $0.18[-1.73,2.09]^{\$}$ & $-0.25[-0.98,0.48]^{\$}$ & $-0.39[-2.49,1.71]^{\$}$ \\
\hline Mixed or multiple ethnic origins & $0.15[-0.52,0.83]$ & $-0.15[-0.59,0.29]$ & $0.10[-0.79,0.98]$ \\
\hline Other ethnicity & $0.21[-0.83,1.25]$ & n. $\mathrm{r}^{\#}$ & $0.09[-1.31,1.50]$ \\
\hline \multicolumn{4}{|l|}{ Employment } \\
\hline Key worker & $0.18[-0.06,0.42]$ & $0.07[-0.06,0.19]$ & $0.15[-0.17,0.46]$ \\
\hline Retired & $0.33[-0.15,0.81]$ & $-0.07[-0.23,0.08]$ & $-0.43[-0.87,0.01]$ \\
\hline Student & $0.59[0.11,1.06]$ & $-0.11[-0.71,0.49]$ & $0.00[-0.56,0.56]$ \\
\hline Unemployed & $1.03[0.65,1.42]$ & $-0.24[-0.59,0.12]$ & $0.54[-0.03,1.11]$ \\
\hline \multicolumn{4}{|l|}{ Time of completion } \\
\hline Survey completion date & $-0.05[-0.16,0.06]$ & $-0.07[-0.16,0.02]$ & $0.45[0.28,0.61]$ \\
\hline
\end{tabular}

${ }^{\#}$ within the NBR sample, only one individual reported Other ethnicity, this effect is not reported here; ${ }^{\$}$ sample sizes for these effects did not meet a priori power criteria (based on combined sample) so effects should be interpreted with caution 
In relation to anxiety symptoms, there was no significant effect of diagnosis of eating disorders or personality disorders in NBR and RAMP, there was no significant effect of OCRDs diagnosis in NBR, and no significant effect of ASD diagnosis in GLAD or NBR. In addition, higher symptoms of anxiety were observed in: i) 16-18 year olds in GLAD and NBR, but not RAMP; and ii) key workers, students and individuals who were unemployed in GLAD but not NBR or RAMP.

Table S8. Individual differences in retrospectively estimated GAD-7 symptom change. Estimates, confidence intervals and significance levels of pre-existing diagnostic and demographic variables.

\begin{tabular}{|c|c|c|c|}
\hline & GLAD & NBR & RAMP \\
\hline Intercept & $4.07[3.37,4.77]$ & $1.22[1.04,1.40]$ & $4.33[3.86,4.80]$ \\
\hline Pre-pandemic GAD-7 & $0.56[0.54,0.58]$ & $0.89[0.87,0.91]$ & $0.62[0.59,0.65]$ \\
\hline \multicolumn{4}{|l|}{ Psychiatric diagnoses } \\
\hline Depressive and anxiety disorder & $1.02[0.39,1.64]$ & $1.11[0.93,1.29]$ & $2.17[1.87,2.47]$ \\
\hline Only depressive disorder & $-0.17[-0.85,0.51]$ & $0.40[0.27,0.53]$ & $0.53[0.20,0.86]$ \\
\hline Only anxiety disorder & $0.76[0.05,1.46]$ & $0.28[0.08,0.48]$ & $1.61[1.22,1.99]$ \\
\hline Eating disorders & $0.71[0.39,1.02]$ & $0.31[0.00,0.63]$ & $0.42[-0.06,0.90]$ \\
\hline OCRDs & $0.42[0.13,0.71]$ & $0.06[-0.34,0.47]$ & $0.65[0.19,1.11]$ \\
\hline Psychotic and bipolar disorder & $0.42[-0.44,1.28]$ & $0.38[-1.16,1.91]$ & $0.35[-0.83,1.53]$ \\
\hline Only psychotic disorder & $0.03[-0.69,0.74]$ & $-0.02[-0.90,0.86]^{\$}$ & $-0.29[-1.47,0.88]$ \\
\hline Only bipolar disorder & $0.10[-0.34,0.54]$ & $0.66[0.01,1.31]^{\$}$ & $0.63[-0.22,1.48]$ \\
\hline PTSD & $0.79[0.51,1.07]$ & $0.50[0.16,0.84]$ & $0.36[-0.06,0.78]$ \\
\hline ASD & $0.58[0.01,1.16]$ & $-0.12[-0.80,0.56]^{\$}$ & $0.92[0.19,1.65]$ \\
\hline ADHD & $0.29[-0.50,1.08]$ & $-0.49[-1.32,0.35]^{\$}$ & $-0.38[-1.28,0.52]$ \\
\hline Personality disorder & $0.66[0.27,1.05]$ & $-0.23[-0.83,0.36]^{\$}$ & $0.43[-0.23,1.09]$ \\
\hline \multicolumn{4}{|l|}{ Gender } \\
\hline Female & $0.43[0.17,0.69]$ & $0.42[0.33,0.51]$ & $0.95[0.65,1.25]$ \\
\hline Non-binary/Self-defined & $-0.23[-0.95,0.48]$ & $0.06[-0.70,0.81]^{\$}$ & $0.68[-0.43,1.79]^{\$}$ \\
\hline \multicolumn{4}{|l|}{ Age } \\
\hline $16-18$ years & $0.70[0.11,1.29]$ & $1.98[0.62,3.33]^{\$}$ & $-0.01[-0.58,0.57]$ \\
\hline $19-25$ years & $0.78[0.45,1.10]$ & $0.85[0.48,1.22]$ & $0.67[0.17,1.16]$ \\
\hline $36-45$ years & $-0.42[-0.71,-0.12]$ & $-0.51[-0.71,-0.31]$ & $-0.44[-0.91,0.04]$ \\
\hline $46-55$ years & $-0.63[-0.92,-0.34]$ & $-0.62[-0.80,-0.44]$ & $-1.08[-1.49,-0.66]$ \\
\hline $56-65$ years & $-1.13[-1.50,-0.77]$ & $-0.83[-1.01,-0.65]$ & $-1.39[-1.79,-0.98]$ \\
\hline $66-70$ years & $-1.63[-2.31,-0.94]$ & $-1.04[-1.26,-0.83]$ & $-1.89[-2.45,-1.33]$ \\
\hline $71-75$ years & $-2.00[-2.88,-1.12]$ & $-1.15[-1.38,-0.92]$ & $-2.31[-2.94,-1.68]$ \\
\hline $76+$ years & $-2.11[-3.61,-0.62]^{\$}$ & $-1.06[-1.36,-0.77]$ & $-3.06[-3.90,-2.23]$ \\
\hline \multicolumn{4}{|l|}{ Ethnicity } \\
\hline Asian or Asian British & $0.11[-0.94,1.15]^{\$}$ & $-0.16[-0.55,0.22]$ & $-0.78[-1.59,0.02]$ \\
\hline Black or Black British & $-0.45[-2.25,1.36]^{\$}$ & $-0.05[-0.72,0.61]^{\$}$ & $-0.89[-2.82,1.04]^{\mathrm{s}}$ \\
\hline Mixed or multiple ethnic origins & $-0.25[-0.88,0.39]$ & $-0.25[-0.65,0.15]$ & $-0.23[-1.04,0.58]$ \\
\hline Other ethnicity & $0.11[-0.88,1.10]$ & n.r . $^{\#}$ & $0.06[-1.22,1.33]$ \\
\hline \multicolumn{4}{|l|}{ Employment } \\
\hline Key worker & $0.41[0.18,0.64]$ & $0.04[-0.07,0.16]$ & $0.10[-0.18,0.39]$ \\
\hline Retired & $0.21[-0.24,0.67]$ & $0.02[-0.12,0.16]$ & $-0.37[-0.77,0.04]$ \\
\hline Student & $0.64[0.19,1.09]$ & $0.23[-0.31,0.77]$ & $-0.29[-0.80,0.22]$ \\
\hline Unemployed & $0.87[0.51,1.23]$ & $-0.17[-0.49,0.15]$ & $-0.06[-0.57,0.46]$ \\
\hline \multicolumn{4}{|l|}{ Time of completion } \\
\hline Survey completion date & $-0.08[-0.19,0.02]$ & $-0.07[-0.15,0.02]$ & $0.16[0.02,0.31]$ \\
\hline
\end{tabular}

\#within the NBR sample, only one individual reported Other ethnicity, this effect is not reported here; ${ }^{\$}$ sample sizes for these effects did not meet a priori power criteria (based on combined sample) so effects should be interpreted with caution 